\title{
Finite Element Simulation and Experimental Validation of Distortion and Cracking Failure Phenomena in Direct Metal Laser Sintering Fabricated Component
}

\author{
Yi Zhang, Jing Zhang* \\ Department of Mechanical Engineering, Indiana University-Purdue University Indianapolis, \\ Indianapolis, Indiana, USA \\ *corresponding author: jz29@iupui.edu
}

\begin{abstract}
A new one-way coupled thermal-mechanical finite element based model of direct metal laser sintering (DMLS) is developed to simulate the process, and predict distortion and cracking failure location in the fabricated components. The model takes into account the layer-by-layer additive manufacturing features, solidification and melting phenomena. The model is first validated using experimental data, then model is applied to a DMLS fabricated component. The study shows how the stress distribution at the support-solid interface is critical to contributing to cracking and distortion. During the DMLS process, thermal stress at the support-solid interface reaches its maximum during the printing process, particularly when the first solid layer is built above the support layer. This result suggests that cracking at the interface may occur during the printing process, which is consistent with experimental observation. Using a design parametric study, a thick and low-density porous layer is found to reduce residual stress and distortion in the built component. The developed finite element model can be used to future design and optimize DMLS process.
\end{abstract}

This is the author's manuscript of the article published in final edited form as: Zhang, Y., \& Zhang, J. (n.d.). Finite Element Simulation and Experimental Validation of Distortion and Cracking Failure Phenomena in Direct Metal Laser Sintering Fabricated Component. Additive Manufacturing. https://doi.org/10.1016/j.addma.2017.05.002 
Keywords: Finite element model; direct metal laser sintering; additive manufacturing; residual stress; distortion

\section{Introduction}

Direct metal laser sintering (DMLS) is a popular additive manufacturing process that fabricates metallic components with layer-by-layer addition of powders. DMLS is sometimes also called metal based selective laser sintering (SLS). In this manufacturing process, the powder bed is selectively melted or sintered by a laser source, and solidified to form a high density component. The DMLS fabricated components usually have a relatively high density of $\sim 95 \%$ with good mechanical strength. High speed moving laser heating leads to a non-uniform distributed temperature field. The non-uniform temperature field, along with hatch overlap, cause a highly inhomogeneous thermal gradient and complicated non-uniform thermal expansion or shrinkage. Therefore, undesired distortion and cracking are often observed on failed printed products. In addition, support materials are needed in the DMLS process to stabilize the structure during printing. The support materials need to be porous so that they are easy to be removed in post processing. Cracks are often observed at the interface between the porous support and solid printed material, due to residual stresses caused by differential coefficient of thermal expansion.

Thermal stress and distortion during selective laser sintering process have been studied previously. Peter et al. experimentally measured the residual stress distribution in a SLS fabricated component and compared with the analytical and finite element solution [1]. Roberts et al. employed the "element birth" method in finite element analysis to simulate the addition of 
material in the SLS process [2]. They simulated the residual stress and distortion of a substrate with simple two-layer structure, and the results were compared with experiment. Paul et al. used the finite element model to predict the shrinkage and distortion of AM fabricated parts with larger component sizes [3]. Heigel et al. developed a coupled thermal-mechanical finite element model. The predicted temperature and deformation results were compared with in-situ experimental measurements [4]. A geometric compensation method was proposed by Senthilkumaran et al. [5]. A better geometric accuracy prediction was obtained when the input geometry was compensated based on simulated thermal deformation and shrinkage results.

Although previous works report stress generation and deformation during DMLS processes, the residual stress at the interface of printed porous support and solid metal part has not been fully understood. Additionally, finite element modeling focusing on stress generation, deformation, crack at the support-solid boundary is rarely reported. In fact, many DMLS components failed due to support-solid interface cracking during the printing process, which dramatically increased the DMLS production costs. Even though some geometry compensation strategies were proposed to overcome the distortion, the cracking problem due to interface stress still exists. The porous support structure has a different coefficient of thermal expansion, and weaker mechanical strength than the solid print, which often lead to crack initiation and propagation at the interface during the printing process even before warping effect occurs.

This paper will develop a new finite element based, thermal-mechanical model including both porous support and solid printed metal components. The stress generation at the supportsolid interface will be focused. The model will be applied to a DMLS printed component, and the predicted distortion and cracking will be validated by experiment. Further, from design perspective, a parametric study with varying densities and thicknesses of the support layer will 
be conducted. The effect of support layer density and thickness on the interface stress will be discussed.

\section{Numerical Method}

\subsection{Governing equations}

One-way coupling of thermal-mechanical model includes both thermal and mechanical components is used in this work. The temperature field is obtained from a transient heat transfer model, and used as the boundary condition of a quasi-static solid mechanical model.

In the heat transfer model, the temperature field under selectively laser heating is obtained from the transient heat conduction equation:

$$
\rho\left(\frac{\partial H}{\partial t}\right)=-\nabla \cdot \boldsymbol{q}+Q
$$

where $\rho$ is the density of material, $H$ is the enthalpy, $t$ is time, $\mathrm{Q}$ is the heat generation rate, and $q$ is the heat flux, which is a function of temperature and thermal conductivity in the 3dimensional Fourier's law:

$$
\boldsymbol{q}=-k \nabla T
$$

where $k$ is the temperature dependent thermal conductivity and $T$ is temperature.

Phase change, including melting and solidification during the laser heating is considered in the heat transfer model. The latent heat is represented in the temperature dependent enthalpy by a sudden increase around the melting temperature. Heat convection is applied at the out surface, according to the Newton's law of cooling: 


$$
\frac{\partial Q}{\partial t}=h A\left(T_{s}-T_{\infty}\right)
$$

where $h$ is the convection coefficient, $A$ is the surface area, $T_{S}$ is the surface temperature and $T_{\infty}$ is the ambient building chamber temperature. In the heat transfer model, for the locations where laser heat source is applied, the radiation phenomenon is neglected $[6,7]$, due to its minor contribution to overall heat transfer in the model.

In the quasi-static solid mechanical model, the 3-dimentional equation of motion is described by Newton's second law:

$$
\mathbf{0}=\nabla \boldsymbol{\sigma}+\boldsymbol{F}_{\boldsymbol{v}}
$$

where $\boldsymbol{\sigma}$ is the Cauchy stress tensor and $\boldsymbol{F}_{\boldsymbol{v}}$ is the body force. The stress tensor $\boldsymbol{\sigma}$ is calculated by 3-dimensional Hook's law:

$$
\boldsymbol{\sigma}=\boldsymbol{D} \cdot \boldsymbol{\varepsilon}
$$

with $\boldsymbol{D}$, the stiffness matrix, and $\boldsymbol{\varepsilon}$, the total strain tensor, which is a combination of three terms [8]:

$$
\varepsilon=\varepsilon_{e l}+\varepsilon_{i e}-\varepsilon_{t h}
$$

where $\varepsilon_{\boldsymbol{e}}$ is the elastic strain, and $\boldsymbol{\varepsilon}_{\boldsymbol{i} \boldsymbol{e}}$ is the inelastic strain calculated from temperaturedependent stress deviation, strain hardening, and plastic yielding. $\boldsymbol{\varepsilon}_{\boldsymbol{t} \boldsymbol{h}}$ is the strain due to thermal expansion, which can be obtained by $\boldsymbol{\varepsilon}_{\boldsymbol{t h}}=\alpha \Delta T \mathbf{1}$ (where $\alpha$ denotes the temperature-dependent coefficient of thermal expansion and $\mathbf{1}$ is the unit tensor).

An important feature of the model is the "element birth and death" which captures the layer-by-layer melting and solidification sintering process. Numerically, in order to maintain a 
constant number of equations of motion, deactivation of elements is realized by multiplying a small factor $\left(1 \times 10^{-9}\right)$ to the stiffness of "dead" elements. Since powders are needed to be added to the simulation domain, all elements except the substrate are "killed" or deactivated in the beginning of simulation, and powders are not considered for mechanical computation (i.e., powders have almost zero stiffness). With the scanning of laser beam, elements with temperatures higher than the melting point are "reborn" or activated and their material properties are changed to the solid phase. Later during printing, depending on temperatures, the reborn elements become solid or liquid phases. The shape change associated with melting process is not considered in this work.

\subsection{Model geometry and boundary conditions}

The geometry of the DMLS components used in the validation experiment is shown in Figure 1a. Since this method needs to capture the laser path at micro meter scale, the actual size of this geometry is not able to be modeled due to the computational costs. Instead the FE model is scaled down to $1 / 40$ of the original size. Also, due to reduced-size model, a few geometric details are omitted in the discretized model. As shown in Figure 1b, the dimension of each block is $100 \times 100 \times 75 \mu \mathrm{m}^{3}$, where $100 \mu \mathrm{m}$ is the effective laser spot size [9] and the $75 \mu \mathrm{m}$ is layer thickness. The porous support layer, solid print component, and printing plate are given in Figure 1b. Each powder layer is represented by two equal sized element layers with a thickness of 37.5 $\mu \mathrm{m}$. 


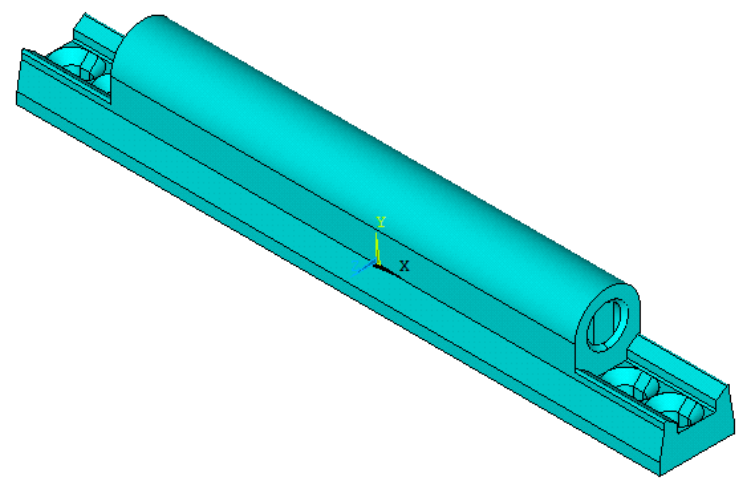

(a)

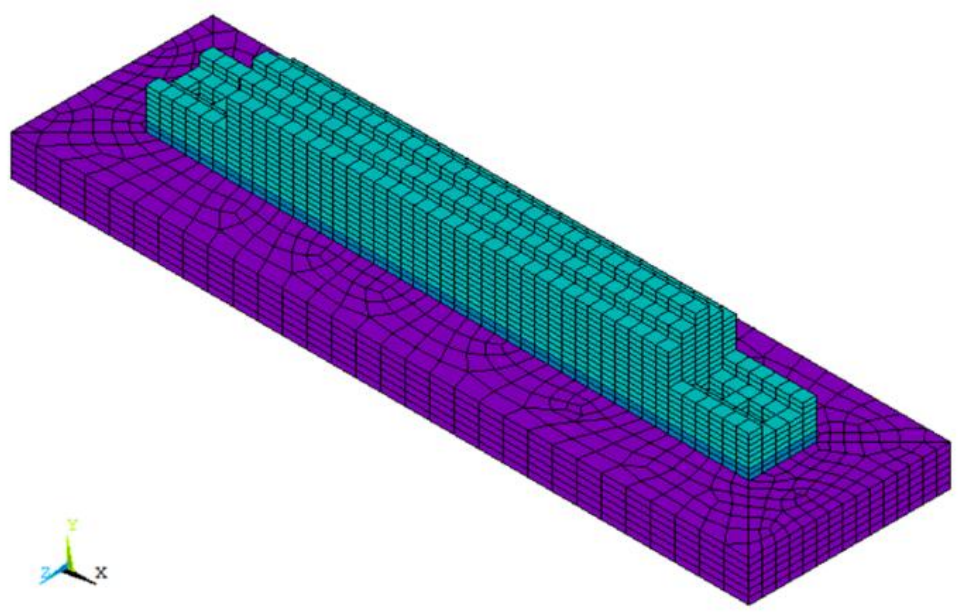

(b)

Figure 1: (a) CAD geometry of the DMLS component (dimension $\mathrm{L} \times \mathrm{W} \times \mathrm{H}$ is $\sim 20 \mathrm{~cm} \times 2 \mathrm{~cm} \times 4$ $\mathrm{cm}$ ); (b) the voxelized 1/40 scaled FE mesh used in the model. The purple elements represent AISI stainless steel plate, dark blue elements show the 15-5 PH1 stainless steel support structure, and the light blue elements represent 15-5 PH1 stainless steel solid part. 
The boundary conditions of both thermal and mechanical models are summarized in Figure 2. In the transient thermal model, laser heat flux with a Gaussian laser irradiance distribution is applied to the surface of a small area $(100 \mu \mathrm{m} \times 100 \mu \mathrm{m})[10]$ :

$$
q=\frac{1}{A} \int_{S} I d A=a \cdot \frac{1}{\pi R^{2}} \int_{0}^{R} \frac{2 P}{\pi R^{2}} e^{-2 r^{2} / R^{2}} \cdot 2 \pi r d r
$$

where $\mathrm{q}$ is the laser heat flux, $\mathrm{P}$ is power, $\mathrm{R}$ is the laser spot radius and $a$ is the material absorptance. In this study the absorptance is taken from Bertolotti [11], which is 0.2 for solid phase and 0.1 for liquid phase of materials.

In Eq. 1, additional terms are included corresponding to preheat convection, $T=T_{0}$, and the surface convection, $(-k \nabla T) \cdot \vec{n}=h\left(T-T_{0}\right)$, where $\mathrm{h}$ is the convection coefficient. The printed component surface and the substrate top surface are free to convection with air. For each layer of scanning, the "s" shape of laser scanning path is applied. Between each layer, free convection of $8 \mathrm{~s}$ is simulated, corresponding to the recoating process. After all the layers are built, the model cools down to room temperature. In the mechanical analysis model, one end of the substrate is fixed to all degree of freedom, thus forming a cantilever support $[2,3,12]$. 


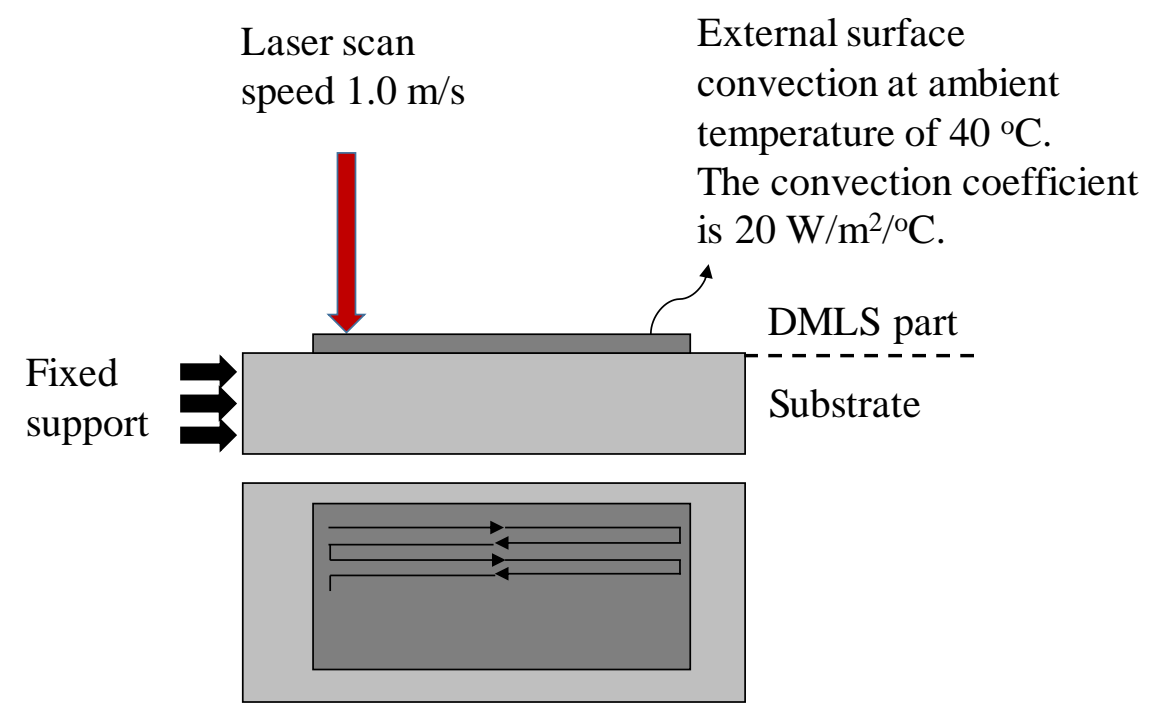

(a)

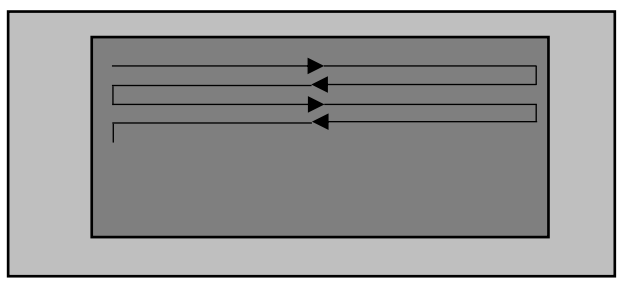

(b)

Figure 2: Thermal and structural boundary conditions in the model (a) side view, (b) top view; it also shows the "s" shape of laser scanning path.

\subsection{Materials properties}

The mechanical and thermal properties of the AISI stainless steel plate are adopted from Ref.[12], since the same substrate material is used. We were not able to find the temperaturedependent material properties of 15-5 PH1 stainless steel in this particular temperature range from the literature, therefore some properties are assumed to be temperature-independent based on Ref.[13], or using the properties of forged 15-5 steel [14]. For the support layer, since it is 
porous and also made of 15-5 stainless steel, the properties, including density, Young's modulus, yield strength, tangent modulus, and coefficient of thermal expansion are scaled by a factor based on relative density. In this work: relative density of $0.4,0.6$ and 0.8 are used in the design parametric study in Section 3.4. Other properties are same as the 15-5 stainless steel solid component. The material properties used the heat transfer simulation and solid mechanical simulation are summarized in Table 1 and Table 2 , respectively.

Table 1: Thermal properties for 15-5 stainless steel [13, 14], and AISI stainless steel [12].

\begin{tabular}{|l|l|l|l|l|}
\hline & \multicolumn{3}{|l|}{$15-5$ stainless steel } & AISI stainless steel \\
\hline $\begin{array}{l}\text { Temperature } \\
\left({ }^{\circ} \mathrm{C}\right)\end{array}$ & $\begin{array}{l}\text { Thermal } \\
\text { conductivity } \\
\left(\mathrm{W} / \mathrm{m} /{ }^{\circ} \mathrm{C}\right)\end{array}$ & $\begin{array}{l}\text { Enthalpy } \\
(\mathrm{kJ} / \mathrm{kg})\end{array}$ & $\begin{array}{l}\text { Thermal conductivity } \\
\left(\mathrm{W} / \mathrm{m} /{ }^{\circ} \mathrm{C}\right)\end{array}$ & $\begin{array}{l}\text { Enthalpy } \\
(\mathrm{kJ} / \mathrm{kg})\end{array}$ \\
\hline 25 & 12.9 & 0 & 51.9 & 0 \\
\hline 400 & 22.5 & 172.5 & 49.9 & 225 \\
\hline 800 & 22.6 & 356.5 & 29.3 & 490 \\
\hline 1640 & 22.6 & 742.9 & 30 & 1340 \\
\hline 1660 & 22.6 & 1032.1 & 30 & 1355 \\
\hline 2800 & 22.6 & 1556.5 & 30 & 1710 \\
\hline
\end{tabular}

Table 2: Mechanical properties of 15-5 [13, 14] and AISI stainless steels [12].

\begin{tabular}{|l|l|l|l|l|l|l|l|}
\hline $\begin{array}{l}15-5 \\
\text { stainless } \\
\text { steel }\end{array}$ & $\begin{array}{l}\text { Temp. } \\
\left({ }^{\circ} \mathrm{C}\right)\end{array}$ & $\begin{array}{l}\text { Density } \\
\left(\mathrm{kg} / \mathrm{m}^{3}\right)\end{array}$ & $\begin{array}{l}\text { Young's } \\
\text { modulus } \\
(\mathrm{GPa})\end{array}$ & $\begin{array}{l}\text { Poisson's } \\
\text { ratio }\end{array}$ & $\begin{array}{l}\text { Coeff. of } \\
\text { thermal } \\
\text { expansion } \\
\left(1 \times 10^{6} /{ }^{\circ} \mathrm{C}\right)\end{array}$ & $\begin{array}{l}\text { Yield } \\
\text { strength } \\
(\mathrm{MPa})\end{array}$ & $\begin{array}{l}\text { Tangent } \\
\text { modulus } \\
(\mathrm{GPa})\end{array}$ \\
\cline { 2 - 8 } & 25 & 4420 & 160 & 0.272 & 11.9 & 1062 & 120 \\
\cline { 2 - 8 } & 200 & 4365 & 140 & 0.272 & 12.4 & 743 & 130 \\
\cline { 2 - 8 } & 315 & 4310 & 131 & 0.272 & 12.8 & 670 & 160 \\
\cline { 2 - 8 } & 540 & 4190 & 80 & 0.38 & 13.1 & 478 & 190 \\
\cline { 2 - 8 } & 2800 & 3140 & 1 & 0.45 & 25 & 255 & 2 \\
\hline \multirow{2}{*}{$\begin{array}{l}\text { AISI } \\
\text { stainless } \\
\text { steel }\end{array}$} & 25 & 7950 & 200 & 0.26 & 10.7 & 178 & 3 \\
\cline { 2 - 8 } & 200 & 7785 & 200 & 0.26 & 13.7 & 178 & 2 \\
\cline { 2 - 8 }
\end{tabular}




\begin{tabular}{|l|l|l|l|l|l|l|l|}
\hline \multirow{2}{*}{} & 540 & 7260 & 147 & 0.38 & 16.2 & 112 & 1 \\
\cline { 2 - 8 } & 2800 & 5630 & 1 & 0.45 & 25 & 1 & 1 \\
\hline
\end{tabular}

\subsection{Simulation analyses}

The simulation is a one-way coupling the following two steps: (1) a transient thermal laser heating analysis to calculate the layer-by-layer addition of powders, powder melting and solidification, and temperature evolutions; and (2) a transient solid mechanical analysis, which uses the temperature distribution data from step (1) as the input to calculate residual stress, deformation and distortion. A user defined macro, including FE mesh, material properties, loads, boundary conditions and solver configurations, is applied in the commercial finite element software package ANSYS Mechanical APDL [15]. The flow chart of the whole FE model is shown in Figure 3.

In the thermal analysis, the laser heat source is applied as a continuous moving inward heat flux. The movement of laser heat flux is realized by changing the heat flux location in the user defined macro. The laser heating path is defined by imposing heat flux to the finite elements along the path. When the laser spot location changes from one element to the next, the thermal analysis results (temperature and heat flux) from the previous location are applied as initial conditions of the next step. The movement of the laser and the deposition of layer ("reborn" of elements) are automatically controlled by the user defined macro.

During the thermal analysis, the temperature field results of each step are recorded. At the completion of thermal analysis, all recorded temperature data are imported to the mechanical model as the initial conditions. Same mesh and time control parameters in the thermal model are applied to the mechanical model. Similar to the thermal analysis, the user macro controls how the melted elements activate or reborn in a discrete way based on the temperature distribution 
along the laser path. Then the thermal and mechanical loads are applied to the activated the elements. The nodal displacement results after previous step are applied to the next step as the initial condition.
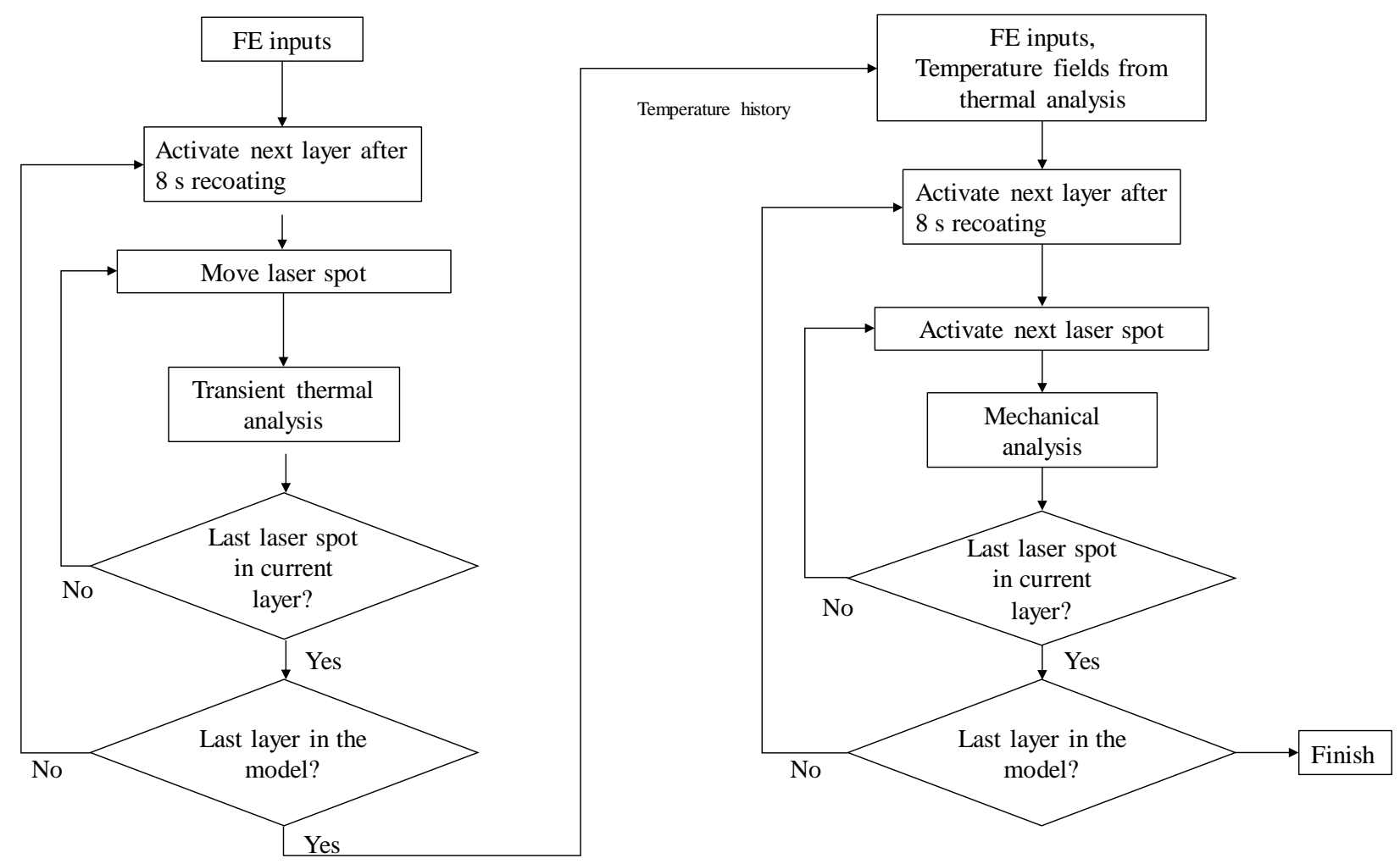

Figure 3: Flow chart of the one-way coupled thermal-mechanical finite element model

\section{Results and discussion}

\subsection{Model validation}

Method used in this work is first validated by using a TiAl6V4 block with the dimension of $1 \mathrm{~mm} \times 2 \mathrm{~mm} \times 60 \mu \mathrm{m}$ printed on a $5 \mathrm{~mm} \times 5 \mathrm{~mm} \times 1 \mathrm{~mm}$ AISI 1015 stainless steel substrate plate (Figure 4b). The simulated substrate deformation (Figure 4a) is compared with experimental data in Ref.[12]. 


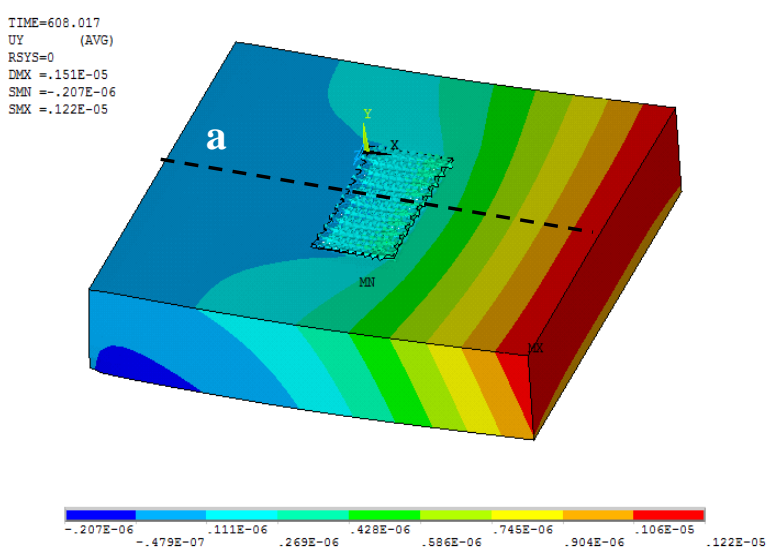

(a)

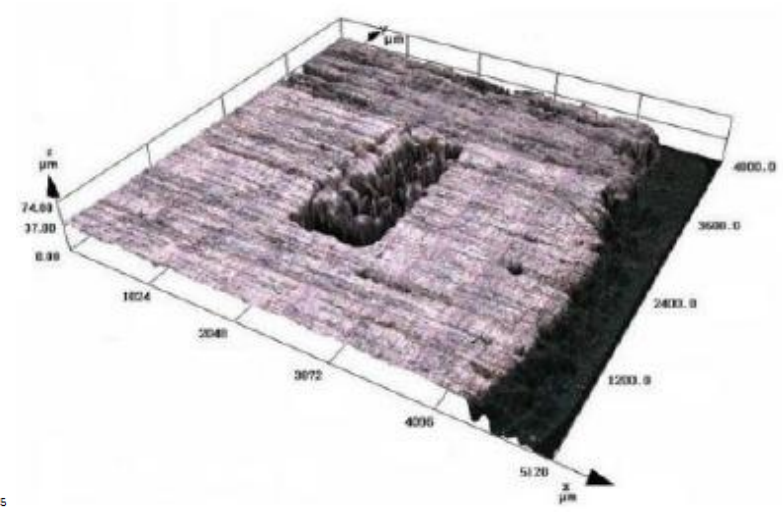

(b)

Figure 4: (a) Simulated deformation in this work, and (b) topology experimental measurements [12].

The deformation along the path "a" on top surface of substrate place in Figure 4a is used to compare with the surface topology experimental measurement. As shown in Figure 5, reasonably good agreement is obtained between the prediction (Figure 5a) and experimental measurement (Figure 5b), where the dashed curves are fitted by measured data points. On both figures, the left-end has zero displacement as a result of the fixed boundary condition. Due to the plastic deformation caused by the thermal expansion, the right-side shows a permeant upward displacement of about $1.3 \mu \mathrm{m}$. 


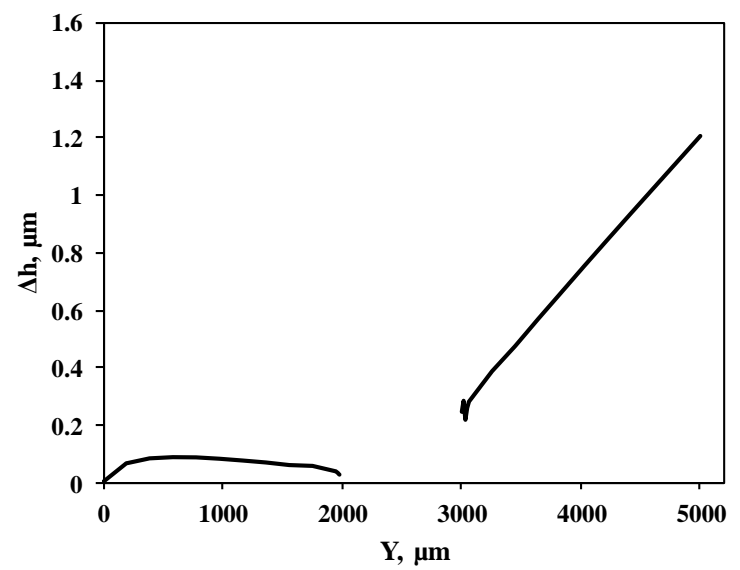

(a)

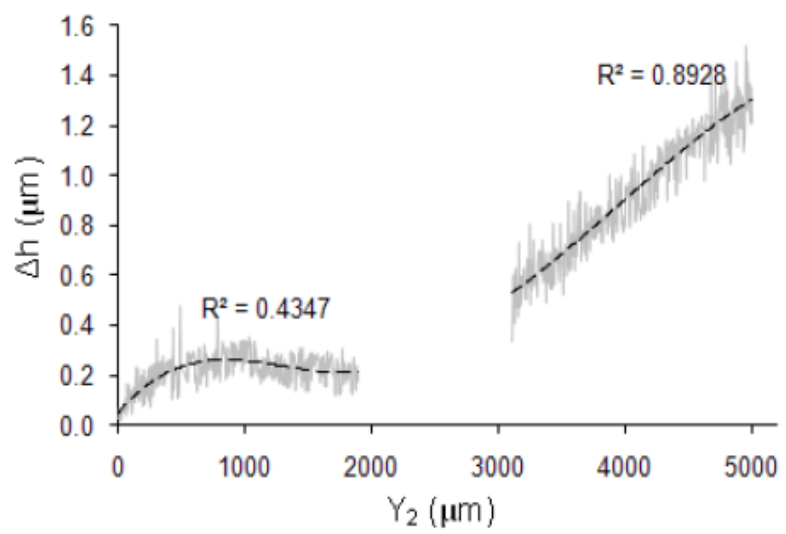

(b)

Figure 5: Surface deformation along path "a" in the validation model in Figure 4a. (a) prediction in this work, (b) experimental measurements [12].

\subsection{Thermal analysis results of the DMLS fabricated component}

After model validation, the DMLS process of 15-5 stainless steel using the geometry shown in Figure $1 \mathrm{~b}$ is simulated. The scaled model has 9 scanning layers with a layer thickness of $75 \mu \mathrm{m}$. The porous support material is assigned to the first layer. A scaling factor of 0.6 of the solid material, estimated from porosity of the porous support layer, is used for the mechanical properties of the support layer. The simulation starts with a transient thermal analysis, and the resulting temperature history is used for a mechanical structural analysis.

The temperature distribution evaluations within one scan layer can be illustrated in Figure 6. The rectangle black lines on the top surface show the DMLS 15-5 stainless steel component foot print boundary on the AISI 1015 stainless steel substrate plate. Outside the boundary, the 15-5 stainless steel powders are presented. As shown in Figure 6, the laser movement paths can be clearly illustrated from the temperature distributions with time. With a laser power of $195 \mathrm{~W}$, 
and scan speed of $100 \mathrm{~mm} / \mathrm{s}$, the maximum temperature at the center of laser spot is in the range of $1800-2050{ }^{\circ} \mathrm{C}$. A concentrated high temperature spot with a "tail" is shown in each instant. All the other scan layers show the similar temperature distribution patterns, since the scan strategies are the same. After all layers are built, the entire model is subjected to free convection for $600 \mathrm{~s}$ with a convection coefficient of $20 \mathrm{~W} / \mathrm{m}^{2} /{ }^{\circ} \mathrm{C}$. The simulation is ended with a uniformly distributed ambient temperature of $40{ }^{\circ} \mathrm{C}$. The resultant temperature history is imported to the time-dependent quasi-static solid mechanical model for residual stress and distortion analyses.
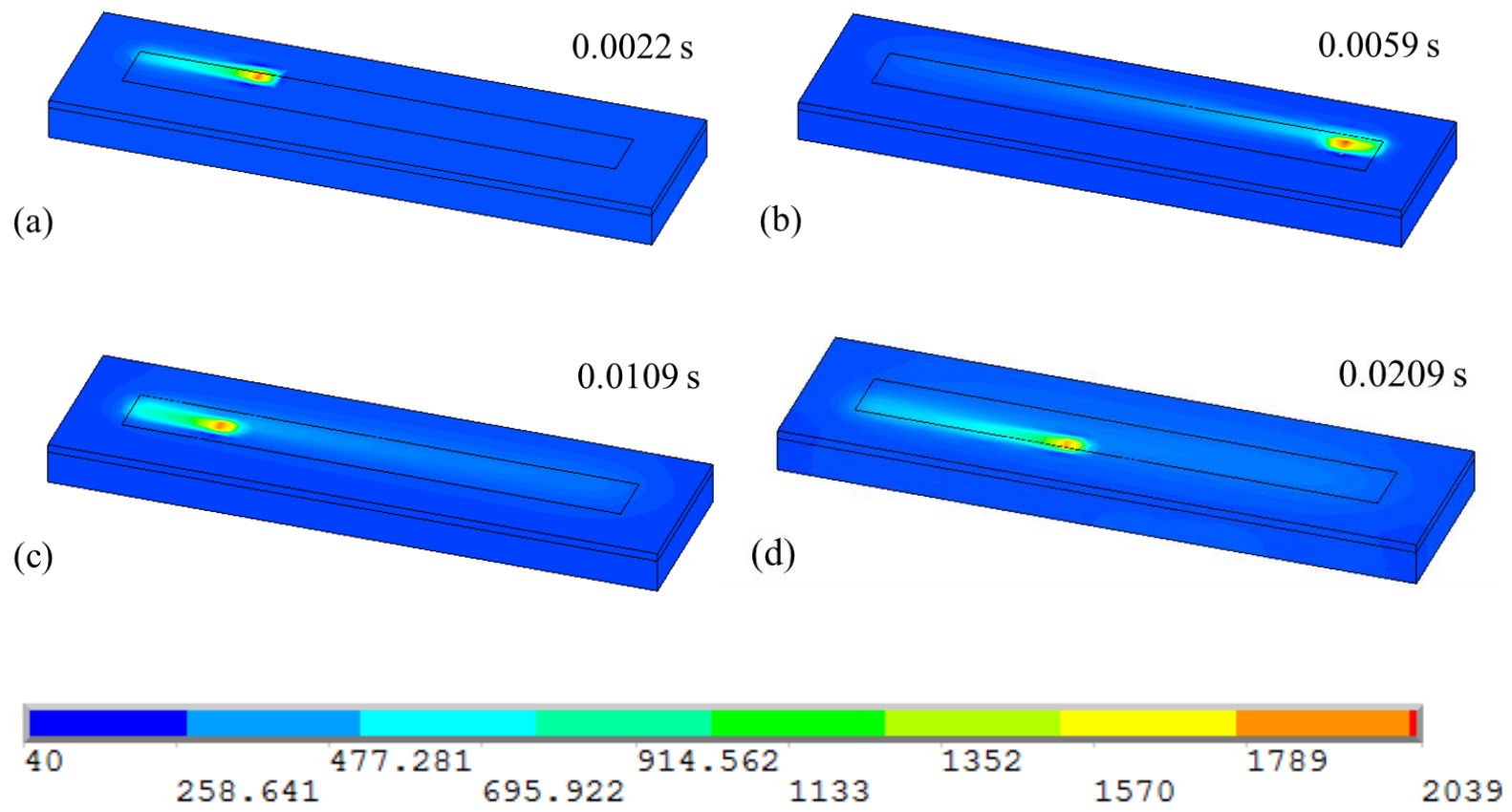

Figure 6: Temperature distribution $\left({ }^{\circ} \mathrm{C}\right)$ of the first layer 15-5 stainless steel sintered on the top of AISI 1015 stainless steel substrate plate at different time.

Although temperature distribution for each layer follows the similar pattern, the magnitudes are different. Figure 7 shows how the temperature evolves during the building of layers, and the average laser spot temperature during the scan of each layer is plotted. The 
temperature increases slightly with the addition of layers from $1975{ }^{\circ} \mathrm{C}$ at the first layer, to 2150 ${ }^{\circ} \mathrm{C}$ at the last layer. The increasing of laser spot temperature is due to insufficient heat dissipation. For the first few layers, a large amount of heat is effectively conducted to the substrate by substrate heat dissipation, therefore the temperature is relatively lower. However, the heat conduction and convection for higher layers are not effective. Since the substrate and the previous built layers have higher temperatures, the conduction path is impacted by the current layer to dissipate heat to the substrate. Similar phenomenon has been reported from experimental work by Peyre et al. [16], where the melt pool size slightly increases with increasing deposition layer during direct laser metal deposition process.

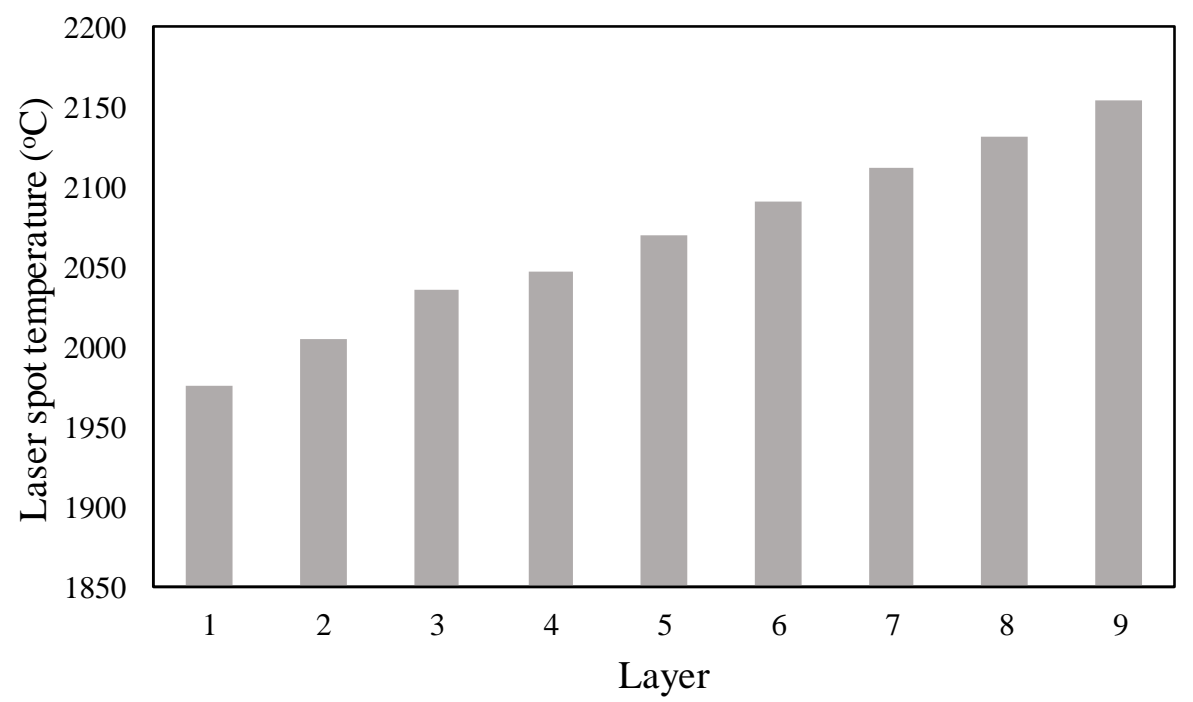

Figure 7: Laser spot temperature evolution with addition of layers. 
3.3 Residual stress and distortion analyses results of the DMLS component

In the solid mechanical model, one end of substrate is fixed and the temperature history is used as thermal boundary condition. Since the surrounding powders have negligible effects on the deformation, the powder elements are omitted in the calculations. The stress, strain, and displacement results are calculated. The von Mises stress of the model after $600 \mathrm{~s}$ cooling process is shown in Figure 8. The top surface shows a high stress due to the discontinuity of voxelized geometry. From this figure, several stress concentration features can be observed: (1) since the substrate is fixed at left end with a cantilever boundary condition, the stress concentration is found at the fixed left surface of substrate; (2) stress concentrations are also found for each printed solid layers, which is a result of high temperature gradient between each layer of elements in the vertical printing direction; and (3) the interface between the porous support layer $\left(1^{\text {st }}\right.$ layer$)$ and the solid component layers $\left(2^{\text {nd }}\right.$ layer and above), which is a combining effect of high temperature gradient and difference of coefficient of thermal expansion. 

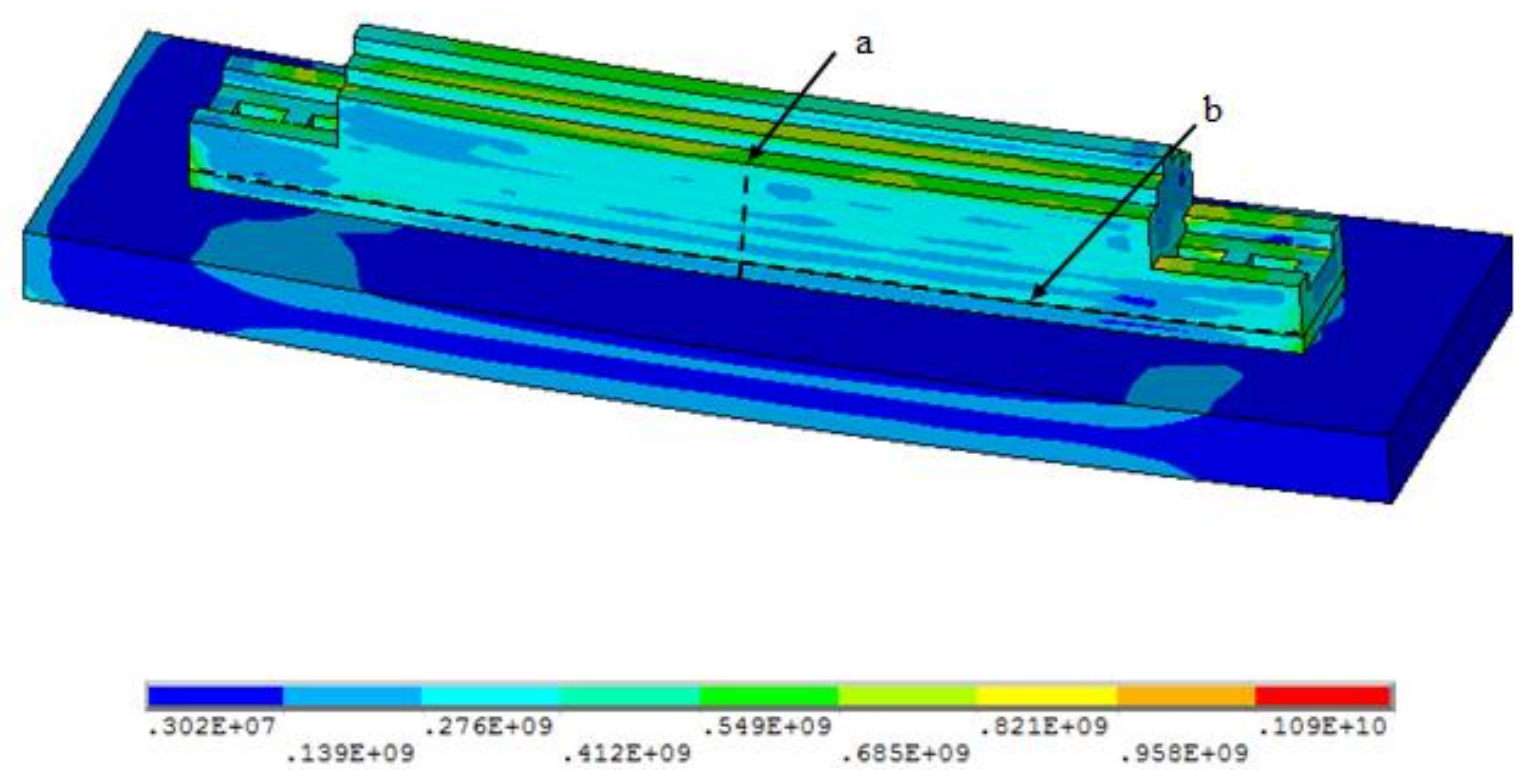

Figure 8: von Mises stress (Pa) distribution of the DMLS 15-5 stainless steel component after cooling.

In order to quantitatively analyze the stress distribution along vertical direction, the residual von Mises stress along path "a" shown in Figure 8 is extracted and presented in Figure 9. In Figure 9, the vertical dash line (height $=75 \mu \mathrm{m}$ ) shows the interface between the porous support layer and the solid component material, and the thin gray vertical lines show the boundary of each layer in the solid part. The stress distribution in solid-solid interface is quite different from the support-solid interface. Between the solid layers, stress continuously increases with the increasing layer height. This is mainly due to the non-uniform thermal gradient induced deformation. The addition of a new layer is actually based on a deformed previous layer, therefore the stress increases. The state of stress of solid layers increases smoothly, and the maximum stress may not able to cause significant failure or damage, since the solid part has high stiffness and yield strength. However, for the porous support layer, due to the thermal expansion difference, a larger stress gradient is observed. As shown in Figure 9, the stress suddenly 
increases from $150 \mathrm{MPa}$ to about $250 \mathrm{MPa}$ at $75 \mu \mathrm{m}$ height, the interface between the support and solid part. Due to its porous structure, the crack resistance (stiffness, yield strength and ultimate strength) of the support material is much lower than the solid part. At the support-solid interface, the locally high stress due to thermal expansion mismatch and temperature gradient can easily reach the yield strength of the porous support material, which could cause failure or crack. Therefore, failure or crack is expected to occur in the support layer close to the supportsolid interface, which is consistent with experimental observations.

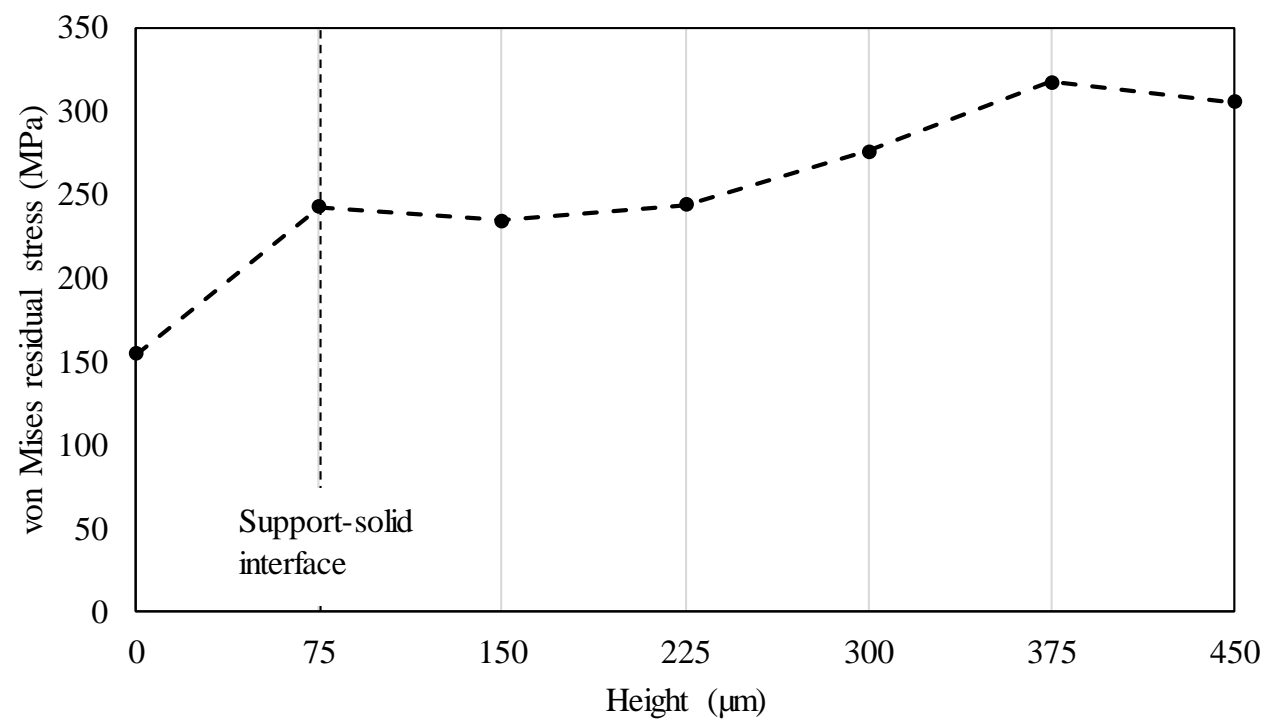

Figure 9: von Mises residual stress along interface between layers (vertical path "a" in Figure 8).

Figure 10 shows the residual stress distribution as the layers are built up. This is done by comparing the von Mises stress distributions along the horizontal path " $b$ " in Figure 8 at different printing time periods without implementing the final stage of cooling by convection. The legend for each curve shows the printing time corresponding to the number of printed layers. In our study, the maximum von Mises stress keeps increasing during the scan of each layer, and 
then it decreases as the recoating process starts. Therefore, the von Mises stresses at the end of each layer are considered as the maximum. As shown in Figure 10, after layer 1 (the support layer) is printed, the interface shows small stresses with a nearly uniform distribution. With the addition of layer 2, thermal stress suddenly increases and reaches to $224 \mathrm{MPa}$. With further addition of layer 3, the stress at interface reduces to about $170 \mathrm{MPa}$. Therefore, the thermally induced residual stress at the support-solid interface reaches to its maximum value when the first solid layer above the support material, layer 2 , is printed. This result suggests that cracking at the interface occurs during the printing process, and it is consistent with the observation from experiment shown in Figure 11a.

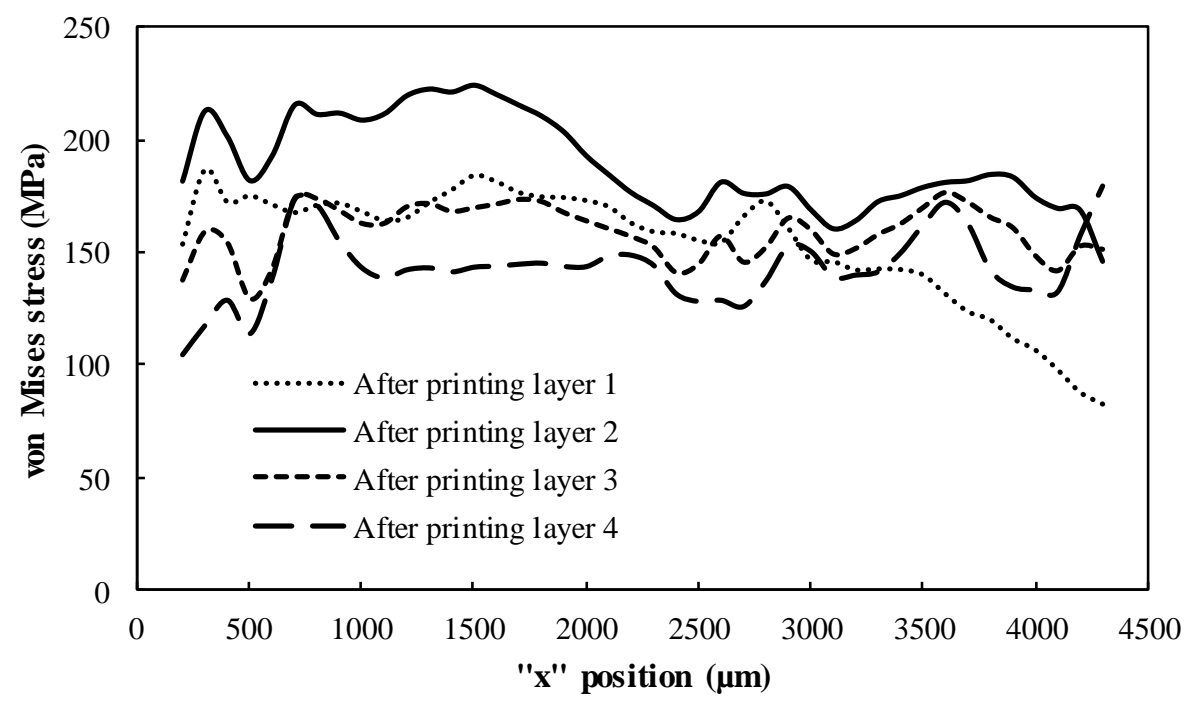

Figure 10: Maximum von Mises stress evolution at the support-solid interface, along the horizontal path "b" defined in Figure 8.

The predicted distortion in printed component is shown in Figure 11a, compared against experiment in Figure 11b. The overall shape distortion comparison shows a good agreement. As 
shown in Figure 11, the distortion is measured at the bottom right of the support material. The predicted distortion from the model is $5.59 \mathrm{~mm}$, which is in good agreement with the experimental measurement of $5.22 \mathrm{~mm}$. The difference between the prediction and experiment can be attributed to model simplification, including deformation relaxation in the experiment. Although the damage criteria are not explicitly considered in this model, the crack location can be estimated by using the maximum von Mises stress. Since von Mises stress is used in yield criteria for metals, and large plastic deformation leads crack initiation in ductile materials. In this work, the cracking location corresponds to a failure $\sim 250 \mathrm{MPa}$ (von Mises) is in the middle of support-solid interface, which is consistent with the previous stress analyses as discussed in Figure 9 and Figure 10.
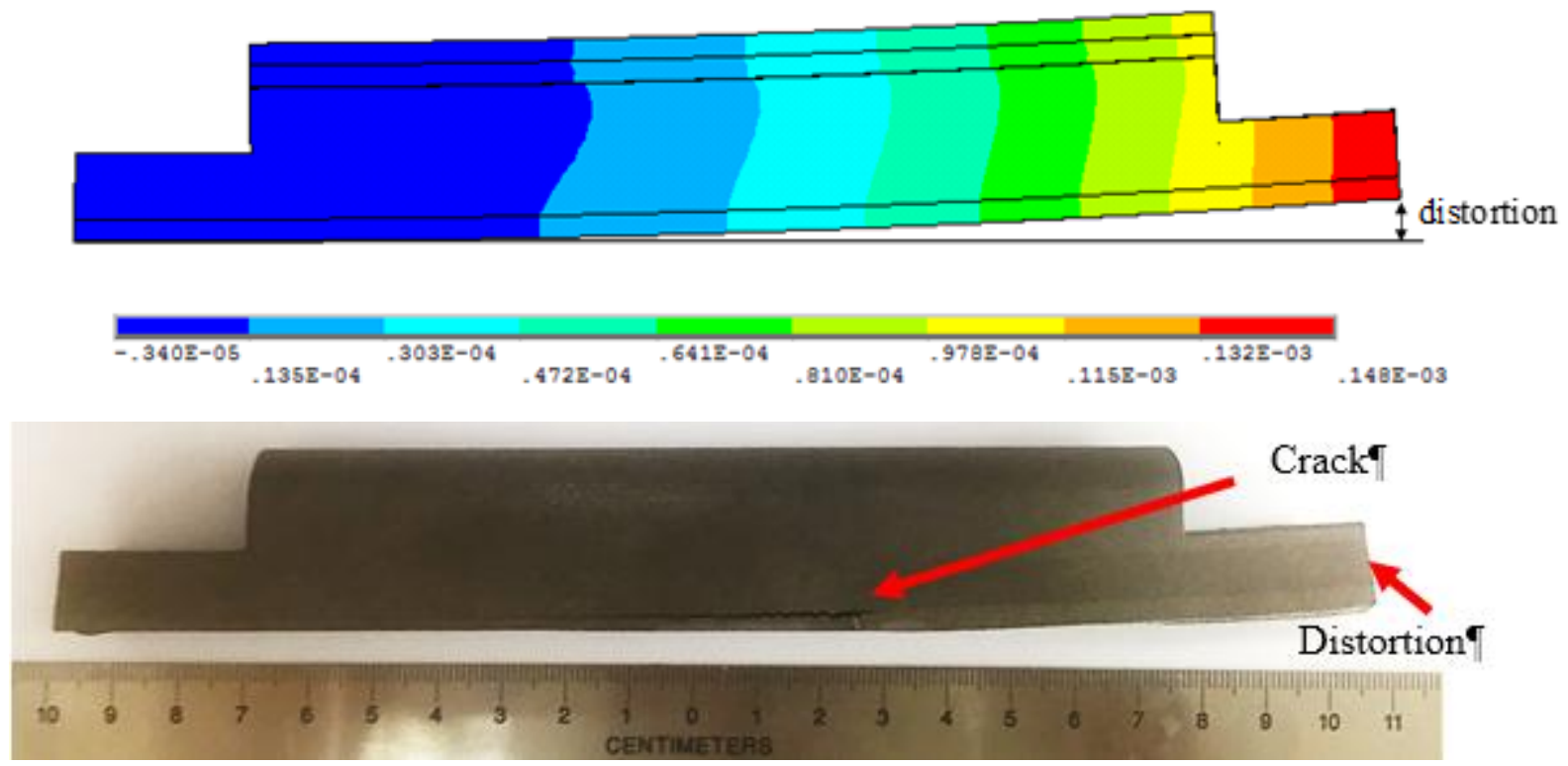

Figure 11: Comparison of simulation predicted distortion with experimental result. Top shows a contour plot of vertical displacement with true scale in meter, and bottom shows the distortion of the DMLS component. The crack and distortion of the component are also marked. 
In addition to final distortion, the evolution of the distortion during the DMLS process is simulated, as shown in Figure 12. Two simulation stages, laser scanning and cooling process, are plotted over time. During the laser scanning stage, the distortion oscillates frequently with time. When the laser heats the top surface of each layer, the top surface has higher temperature than the bottom, so that it expands more and bends the component downward, therefore, a small negative distortion at the free end is shown at the beginning of the scanning (right after $0.001 \mathrm{~s}$ ). After the heat is conducted through the substrate, the previous high-temperature regions cool down, the distortion of the component partially reduces back. However, since the plastic deformation remains, resulting in a positive permanent distortion. Therefore, during the laser scanning stage, the distortion follows a similar oscillating pattern, where a decrease of distortion is due to non-uniform thermal expansion, followed by an increase of accumulated distortion due to plastic deformation. During the cooling stage, or after the DMLS process, no laser heat source is applied, so the distortion changes smoothly. As the cooling begins, the heat conducts from the laser heat region to its surrounding environment, so that the component remains a high temperature that can induce plastic deformation, therefore the distortion keeps increasing (0.2-10 s). When the time is long enough, heat is eventually conducted away, and the temperature drops down. Only shrinkage contributes to the deformation, therefore the distortion decreases after $10 \mathrm{~s}$ until it reaches to a constant value at the ambient temperature. Similar pattern of distortion evolution, i.e., oscillation during the printing and short increase during the cooling, is observed in experiment reported by Denlinger et al. [17]. 


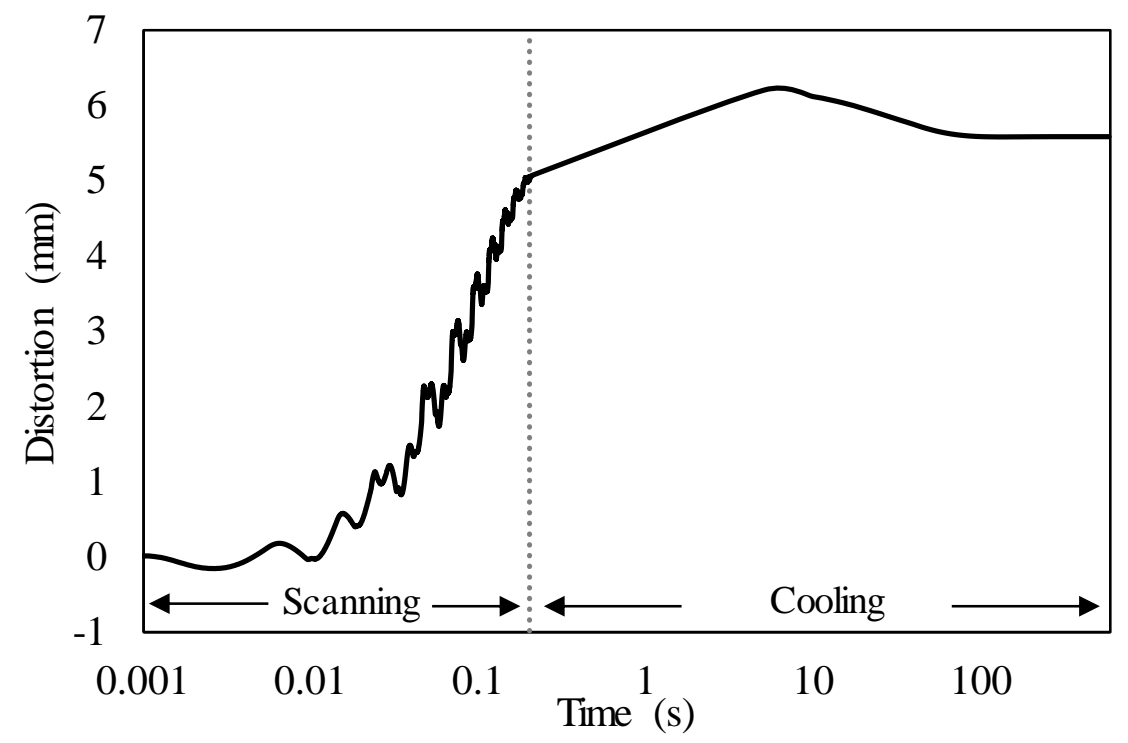

Figure 12: Distortion changes with respect to time. The left side of dashed line shows the laser scanning stage, and the right side shows the cooling stage after DMLS process.

\subsection{Design parametric study results}

From the design point of view, the distortion and cracking in DMLS components should be minimized. In this parametric study, both thickness and density of the support layer are considered. Thicknesses of $75 \mu \mathrm{m}$ and $150 \mu \mathrm{m}$ are chosen with the relative densities of $0.4,0.6$ and 0.8. Therefore, total six simulations cases are conducted.

The resulting maximum von Mises stresses during the printing process with varying parameters are summarized in Figure 13. As shown in the figure, a large support thickness decreases the maximum thermal stress, and the maximum stress increases linearly with relative density. 


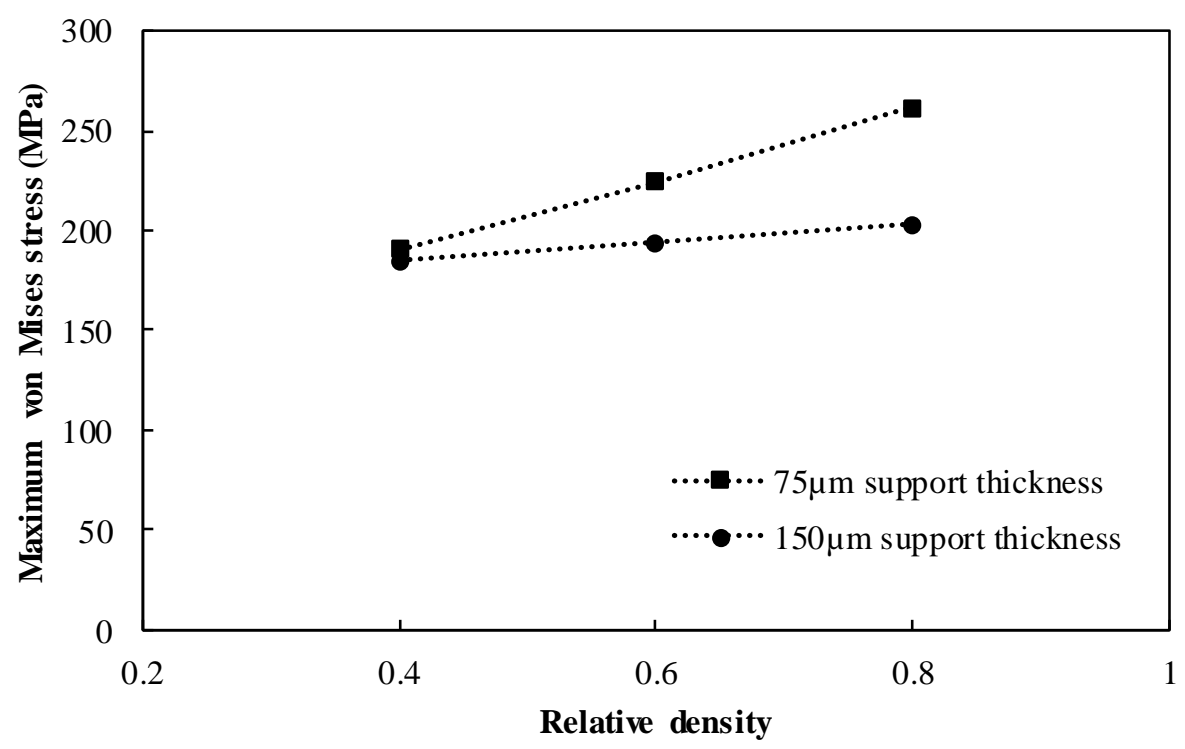

Figure 13: Maximum von Mises stress during the printing as a function of support thickness and relative density.

The distortion results are shown in Figure 14. Distortion decreases with the increasing support layer thickness due to its smaller bending moment. The distortion increases with increasing support relative density, which can also be explained from the stress trends. In summary, a thick and low-density porous layer will reduce the residual stress and distortion in the built component. 


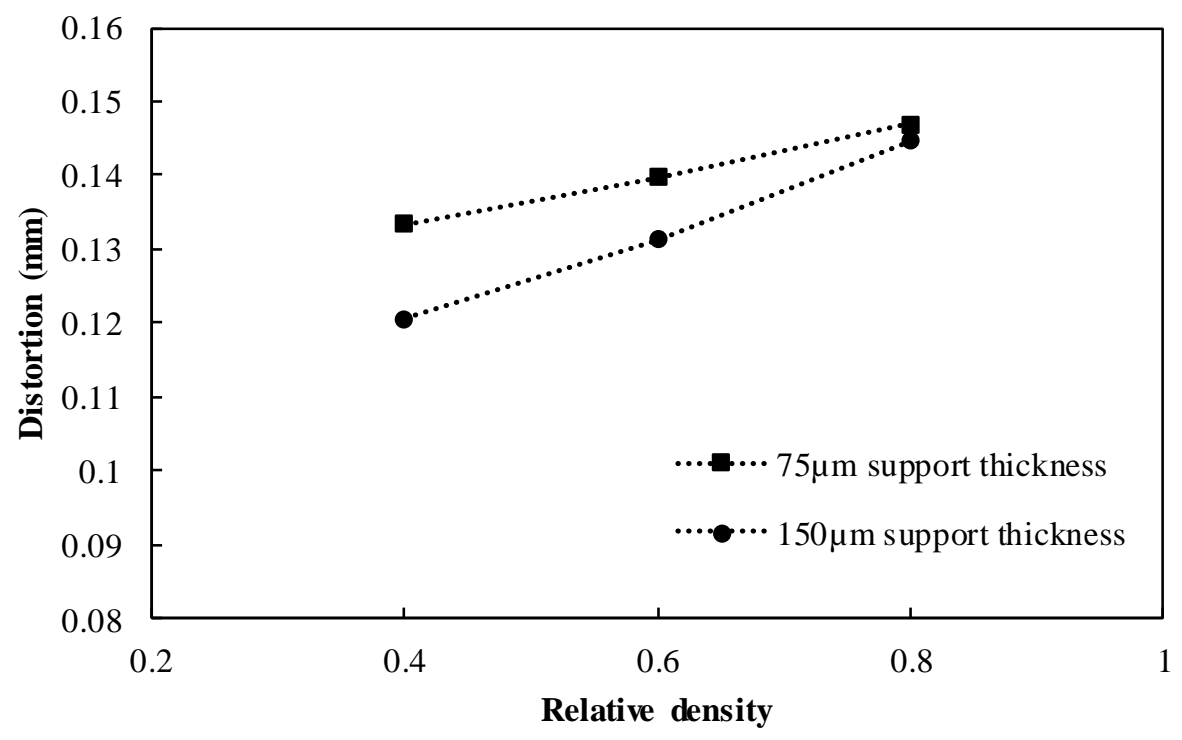

Figure 14: Distortions as a function of support thickness and relative density.

\section{Conclusions}

A new one-way coupled thermal-mechanical finite element model is developed to simulate the DMLS process and predict the distortion and cracking in the fabricated DMLS components. The stress distribution at the support-solid interface and corresponding distortion of DMLS fabricated component are investigated. The main results are summarized as follows:

1. During DMLS process, thermal stress at the support-solid interface reaches its maximum during the printing process, particularly when the first solid layer is built above the support layer. The interface stress drops to a stable value when additional layers are built. This result indicates that cracking may occur at the interface during the printing process, which is consistent with experimental observation. The model successfully predicts the cracking location. 
2. The predicted distortion of the DMLS printed component is in good agreement with experiment.

3. A design parametric study considering the effect of support layer thickness and porosity on the stress and distortion is conducted. The results show that a thick and low-density porous layer will minimize the residual stress and distortion in the built component.

Although the success of current model which is capable to predict the distortion and cracking of DMLS components, it is worthy to note a few limitations in the current model which can serve as the topics of future studies: (1) This work is based on the assumption that there is no relaxation during AM process. If it is considered, the predicted distortion will be slightly less than currently presented. The modeling of deformation relaxation can be included in future models. (2) Due to coupling and layer-by-layer nature of the DMLS process, in order to reduce the computational cost, a dynamic mesh method could be applied. A globally coarse mesh can be assigned, and the mesh is locally refined near the moving laser spot region within each step.

\section{Acknowledgement}

JZ acknowledges the financial support provided by Walmart Foundation (project title: Optimal Plastic Injection Molding Tooling Design and Production through Advanced Additive Manufacturing). Authors also thank the critical comments from two anonymous reviewers. 


\section{References}

1. Mercelis, P. and J.P. Kruth, Residual stresses in selective laser sintering and selective laser melting. Rapid Prototyping Journal, 2006. 12(5): p. 254-265.

2. Roberts, I.A., et al., Numerical and Experimental Studies on the Laser Melting of Steel Plate Surfaces, in Proceedings of the 36th International MATADOR Conference, S. Hinduja and L. Li, Editors. 2010, Springer London: London. p. 535-538.

3. Paul, R., S. Anand, and F. Gerner, Effect of Thermal Deformation on Part Errors in Metal Powder Based Additive Manufacturing Processes. Journal of Manufacturing Science and Engineering, 2014. 136(3): p. 031009-031009.

4. Heigel, J.C., P. Michaleris, and E.W. Reutzel, Thermo-mechanical model development and validation of directed energy deposition additive manufacturing of Ti-6Al-4V. Additive Manufacturing, 2015. 5: p. 9-19.

5. Senthilkumaran, K., P.M. Pandey, and P.V.M. Rao, New model for shrinkage compensation in selective laser sintering. Virtual and Physical Prototyping, 2009. 4(2): p. 49-62.

6. Foroozmehr, A., M. Badrossamay, and E. Foroozmehr, Finite element simulation of selective laser melting process considering optical penetration depth of laser in powder bed. Materials \& Design, 2016. 89: p. 255-263.

7. Streek, A., P. Regenfuss, and H. Exner, Fundamentals of Energy Conversion and Dissipation in Powder Layers during Laser Micro Sintering. Physics Procedia, 2013. 41: p. 858-869.

8. Masubuchi, K. and D.W. Hopkins, Analysis of Welded Structures: Residual Stresses, Distortion, and Their Consequences. 2013: Elsevier Science.

9. Wang, X., Calibration of shrinkage and beam offset in SLS process. Rapid Prototyping Journal, 1999. 5(3): p. 129-133.

10. Shi, Y., et al., Temperature gradient mechanism in laser forming of thin plates. Optics \& Laser Technology, 2007. 39(4): p. 858-863.

11. Bertolotti, M., Physical Processes in Laser-Materials Interactions. 1983: Plenum Press.

12. Roberts, I.A., Investigation of residual stresses in the laser melting of metal powders in additive layer manufacturing. 2012, Ph.D. thesis, University of Wolverhampton.

13. Zeng, P., et al., Nanoparticle sintering simulations. Materials Science and Engineering A, 1998. 252(2): p. 301-306.

14. 15-5 stainless steel data sheet. AK Steel 2007 [cited 2017 Februrary 2nd]; Available from: http://www.aksteel.com/pdf/markets_products/stainless/precipitation/155_ph_data_sheet.pdf.

15. Cole, D.M. and G.D. Durell, The cyclic loading of saline ice. Philosophical Magazine A (Physics of Condensed Matter, Defects and Mechanical Properties), 1995. 72(1): p. 209229.

16. Peyre, P., et al., Analytical and numerical modelling of the direct metal deposition laser process. Journal of Physics D: Applied Physics, 2008. 41(2): p. 025403.

17. Denlinger, E.R. and P. Michaleris, Effect of stress relaxation on distortion in additive manufacturing process modeling. Additive Manufacturing, 2016. 12, Part A: p. 51-59. 